BULLETIN OF THE

AMERICAN MATHEMATICAL SOCIETY

Volume 77, Number 1, January 1971

\title{
THE REDUCIBILITY OF THOM COMPLEXES AND SURGERY ON MAPS OF DEGREE $d$
}

\author{
BY MAX K. AGOSTON
}

Communicated by N. E. Steenrod, July 27, 1970

1. Statement of results. In this paper we announce a substantial extension of the obstruction theory developed in [1]. Detailed proofs and further properties will appear elsewhere. Basically what we have accomplished is to put the theory in [1] in a more natural setting and extend it to the metastable range. We also apply it to the question of when the Thom complex $T(\xi)$ of a vector bundle $\xi$ over a manifold is reducible and hence get an obstruction theory for imbedding 1-connected manifolds in the metastable range.

All manifolds will be oriented, compact, and differentiable. Bundles are also assumed to be oriented. Let $\theta^{a}$ denote the trivial $q$-plane bundle over a space and set $\pi_{r, t}=\pi_{r} S^{r-t}$.

TheOREM 1 (The Reducibility TheOREM). Let $\xi^{k}$ be $a k$-plane bundle over a closed connected manifold $M^{n}$ and assume $2 k \geqq n+3$. Then

(a) The Thom complex $T\left(\xi \oplus \theta^{1}\right)$ is reducible whenever a sequence of obstructions $o_{i}(\xi) \in H^{i}\left(M ; \pi_{n+k, i-1}\right), 1<i \leqq n$, vanishes. In the stable range, $k \geqq n+3$, the converse is also true, namely, the reducibility of $T\left(\xi \oplus \theta^{1}\right)$ implies the vanishing of the obstructions $o_{i}(\xi)$.

(b) Let $s_{*}: H^{i}\left(M ; \pi_{n+k, i-1}\right) \rightarrow H^{i}\left(M ; \pi_{n+k+1, i-1}\right)$ be the homomorphism induced by the suspension homomorphism $\pi_{n+k, i-1} \rightarrow \pi_{n+k+1, i-1}$. Then $s_{*}\left(o_{i}(\xi)\right)=o_{i}\left(\xi \oplus \theta^{1}\right)$.

Next, let $G_{r, t}$ denote the kernel of the suspension homomorphism $\pi_{r, t} \rightarrow \pi_{l}^{s}=\lim _{q \rightarrow \infty} \pi_{r+q, t}$ and let $G_{r, t}^{q}$, for $q \leqq r$, be the subgroup of those elements in $G_{r, t}$ which lie in the image of the suspension homomorphism $\pi_{q, t} \rightarrow \pi_{r, t}$. Then $G_{r, t}^{q} \subseteq G_{r, t} \subseteq \pi_{r, t}$.

Combining Theorem 1 with [6], we get:

Theorem 2 (The IMbedding theorem). Let $M^{n}$ be a 1 -connected closed manifold that immerses in $S^{n+k}$ with normal bundle $\xi^{k}$, where $n \geqq 5$

AMS 1970 subject classifications. Primary 55G35, 57D65; Secondary 57D40.

Key words and phrases. Thom complexes, reducibility, obstructions, imbeddings, $\pi$-manifolds, metastable range, surgery, maps of degree $d$, splittings, complete splittings.

Copyright (0) 1971. American Mathematical Society 
and $2 k \geqq n+3$. Assume $n \neq 2(\bmod 4)$. Then $M^{n}$ imbeds in $S^{n+k+1}$ with normal bundle which is fiber homotopy equivalent to $\xi^{k} \oplus \theta^{1}$ provided that a sequence of obstructions $o_{i}(\xi) \in H^{i}\left(M ; \pi_{n+k, i-1}\right), 1<i \leqq n$, vanishes. The $o_{i}(\xi)$ lie in the image of the map $H^{i}\left(M ; G_{n+k, i-1}\right) \rightarrow H^{i}\left(M ; \pi_{n+k, i-1}\right)$. If $M$ is a $\pi$-manifold, then the $o_{i}(\xi)$ lie in the image of the map $H^{i}\left(M ; G_{n+k, i-1}^{n}\right) \rightarrow H^{i}\left(M ; \pi_{n+k, i-1}\right)$.

Theorem 2 has application to manifolds which have only finite homology groups (except in dimension 0 and $n$ ). Furthermore, consider the following hypothesis:

$$
G_{n+k, i-1}^{n}=0 \text { whenever } 2 k \geqq n+3 \text { and } 1<i \leqq n .
$$

Theorem 2 and $\left(^{*}\right)$ implies that all 1 -connected $\pi$-manifolds $M^{n}$, $n \geqq 5$, imbed in essentially the metastable range. Although $\left(^{*}\right)$ seems plausible, its truth or falsity is unknown.

The proofs of Theorems 1 and 2 can be used to prove some nonimbedding theorems by showing that certain obstructions are always nonzero and that none of the normal bundles for any immersion are reducible.

Before we can state the next theorem, which is really about surgery on maps of degree $d$ and from which Theorem 1 will follow, we need some notation.

Definition. Let $x \in \pi_{n+k} T(\xi)$. We say that $x$ splits if, for some integer $t>0, x$ can be represented by a map $F: S^{n+k} \rightarrow T(\xi)$, transverse regular on $M$, such that

(i) $F^{-1}(M)$ has $t$ components, $N_{1}, N_{2}, \cdots, N_{t}$; and

(ii) $F \mid N_{i}: N_{i} \rightarrow M$ has degree \pm 1 .

$F$ is called a splitting of $x$. We say that $x$ splits completely if, in addition to (i) and (ii), the splitting can be chosen so that it satisfies

(iii) $F \mid N_{i}$ is a homotopy equivalence.

Note. Let $\operatorname{deg}: \pi_{n+k} T(\xi) \rightarrow H_{n+k} T(\xi)$ denote the Hurewicz homomorphism. (It is easily seen that, with the proper choice of orientations, deg $x$ is the degree of $F \mid F^{-1}(M): F^{-1}(M) \rightarrow M$, where $F: S^{n+k}$ $\rightarrow T(\xi)$ represents $x$ and is transverse regular on $M$.) The interesting case in the definition is the case $t=|\operatorname{deg} x|>0$. $t$ is allowed to be arbitrary only so that we do not have to make a special case of $\operatorname{deg} x=0$.

TheOREM 3 (The SURgery THEOREM For MAPS OF DEgReE $d$ ). Let $\xi^{k}$ be a $k$-plane bundle over a closed connected manifold $M^{n}$ and assume that $2 k \geqq n+3$. Suppose that $x \in \pi_{n+k} T(\xi)$ and let $s_{\#}: \pi_{n+k} T(\xi)$ $\rightarrow \pi_{n+k+1} T\left(\xi \oplus \theta^{1}\right)$ denote the natural suspension homomorphism. Then

(a) $s_{p}(x)$ splits provided that a sequence of obstructions $o_{i}(\xi)$ 
$\in H^{i}\left(M ; \pi_{n+k, i-1}\right), 1<i \leqq n$, vanishes. The converse is also true if $k \geqq n+3$, namely, if $s_{\#}(x)$ splits, then the obstructions $o_{i}(\xi)$ vanish.

(b) $s_{*}\left(o_{i}(x)\right)=o_{i}\left(s_{\#}(x)\right)$, where $s_{*}$ is the map defined in Theorem 1.b.

Theorem 3(a) and the standard Browder-Novikov theory for doing surgery on maps of degree \pm 1 gives us:

COROLlARY 3.1. In addition to the hypothesis of Theorem 3 assume that $M^{n}$ is 1 -connected, $n \geqq 5$, and $n \neq 2(\bmod 4)$. Then $s_{\sharp}(x)$ splits completely provided that the sequence of obstructions $o_{i}(\xi), 1<i \leqq n$, vanishes. If $n$ is odd, $n \geqq 6$, and the obstructions $o_{i}(\xi)$ only vanish for $1<i<n-1$, then $s_{\#}(x)$ admits a representative $F: S^{n+k+1} \rightarrow T\left(\xi \oplus \theta^{1}\right)$ such that $F$ is transverse regular on $M$ and $F^{-1}(M)$ has the homotopy type of the $t$-fold connected sum of $M$ or $-M$, where $t=|\operatorname{deg} x|$ if $\operatorname{deg} x \neq 0$ and $t=2$ if $\operatorname{deg} x=0$.

Note. In the stable range $k \geqq n+3$, the obvious converse to Corollary 3.1 is also true.

As was observed in [1], Corollary 3.1 can be rephrased to answer the following question which makes explicit the relation to the standard Browder-Novikov theory: Given a normal map $f: V^{n} \rightarrow M^{n}$ of degree $d \neq 0$, when can we do surgery on $V$ to obtain a manifold which is homotopy equivalent either to the $|d|$-fold connected sum of $M$ or to $|d|$ disjoint copies of $M$ ?

2. Outline of proofs. We begin with a discussion of the proof of Theorem 3. The basic idea is already contained in [1], but in a needlessly complicated form. Let us fix a nice Morse function $\rho: M^{n} \rightarrow[0, n]$ (see $[8, \mathrm{p} .44]$ ) and set $M(i)=\rho^{-1}[0, i+1 / 2]$. Then $M(i)$ is obtained from $M(i-1)$ by attaching a certain number of thickened $i$-handles, that is,

$$
\begin{aligned}
M(i)= & M(i-1) \cup(\partial M(i-1) \times[0,1]) \\
& \cup\left(D^{i} \times D^{n-i}\right)_{1} \cup \ldots \cup\left(D^{i} \times D^{n-i}\right)_{n(i)} .
\end{aligned}
$$

Let $x \in \pi_{n+k} T(\xi)$ and choose a representative $F: S^{n+k} \rightarrow T(\xi)$ for $x$ which is transverse regular as needed on various parts of $M$. Set

$$
V^{n}=F^{-1}(M) \text { and } A_{i}=F^{-1}(M(i) \cup \partial M(i) \times[0,1]) .
$$

Our object is to split off a manifold from $V$ on which $F$ will have degree \pm 1 and then to repeat the process to get as many such pieces as desired. In [1] we tried to split off all pieces simultaneously, but this is an unnecessary complication. We assume inductively that $A_{q-1}, 1 \leqq q \leqq n$, has two components $C_{1}$ and $C_{2}$ such that $f \mid C_{1}:\left(C_{1}, \partial C_{1}\right)$ $\rightarrow(M(q-1), \partial M(q-1))$ has degree \pm 1 . Unfortunately, $A_{q}$ may have 
only one component, and therefore we want to cut $A_{q}$ into two components by surgery, extending $C_{1}$ and $C_{2}$. We consider $N_{i}^{a-1}$ $=F^{-1}\left(\partial D^{q} \times 0\right)_{i}$. Then $N_{i}^{q-1}$ is a framed submanifold in $S^{n+k}$, where the framing of $N_{i}^{q-1}$ is the pullback under $F$ of the natural framing of $\left(\partial D^{a} \times 0\right)_{i}$ in $M$ and the restriction to $\left(\partial D^{a} \times 0\right)_{i}$ of a framing of $\xi \mid\left(D^{q} \times 0\right)_{i}$. Thus, $N_{i}^{q-1}$ determines an element of $\pi_{n+k, q-1}$, and this induces a cochain

$$
c_{q}(x) \in C^{q}\left(M ; \pi_{n+k, q-1}\right)=\operatorname{Hom}\left(H_{q}(M(q), M(q-1)), \pi_{n+k, q-1}\right),
$$

where $c_{q}(x)$ is given on the generators $\left(D^{q} \times 0\right)_{i}$ of $H_{q}(M(q), M(q-1))$ by : $c_{q}(x)\left(D^{q} \times 0\right)_{i}$ is that element in $\pi_{n+k, q-1}$ determined by the framed $N_{i}^{q-1}$. One proves that $c_{q}(x)$ is a well defined cocycle and hence determines a cohomology class $o_{q}(x)$.

NoTE. If $V$ is a $\pi$-manifold, then one can show by doing surgery on $V$ in dimensions $\leqq[n / 2]-1$ that $V-A_{t}$ is contained in an $n$-disk in $S^{n+k}$, where $t=[n / 2]+1$. One can show in addition that the framing induced by $F$ on $N_{i}^{q-1}$ is the $k$-fold suspension of a framing of $N_{i}^{q-1}$ is the $n$-disk. Thus, $N_{i}^{q-1}$ determines an element of $\pi_{n+k, q-1}$ which is the suspension of an element in $\pi_{n, q-1}$. This fact is needed in the proof of the last part of Theorem 2.

To show that $o_{q}(x)$ defines the appropriate obstruction theory, one has to define a difference cochain and prove a series of lemmas similar to those in [1]. The basic fact is that if $c_{q}(x)=0$, then one can extend the splitting of $A_{q-1}$ to a splitting of $A_{q}$ by taking the framed manifold whose boundary is $N_{i}^{q-1}$ and using it to cut $F^{-1}\left(D^{q} \times D^{n-q}\right)_{i}$ into two components. (Of course, we shall have to change the map $F$.) This is proved like in [1] using [4]. The only important difference is that we are no longer in the stable range and we have to worry about whether the surgery we want to perform will intersect $M$. This is why we must work in $S^{n+k+1}$. By being careful and keeping $V-A_{q}$ contained in $S^{n+k}$ (although the $A_{q}$ part may move into $S^{n+k+1}$ in a way which we can control), we can prevent intersections and inductively do the surgery.

Finally, one proves that $o_{q}(x)$ depends only on $\xi$ and on nothing else. Since $x=y+(x-y), x$ somehow "contains" every other element; and therefore, if one splits, then they all split. We let $o_{q}(\xi)=o_{q}(x)$. This completes our rough outline of the proof of Theorem 3 .

Theorem 1 follows immediately from Theorem 3 and the observation that the reducibility of $T\left(\xi \oplus \theta^{1}\right)$ is equivalent to finding a $y \in \pi_{n+k+1} T\left(\xi \oplus \theta^{1}\right)$ such that deg $y= \pm 1$. After all, Theorem 3 was an obstruction theory to finding such a $y$. Theorem 2 is a consequence of Theorem 1 and the note following the definition of $c_{q}(x)$. 


\section{REFERENCES}

1. M. K. Agoston, Browder-Novikov theory for maps of degree $d>1$. I, Topology 9 (3) (1970), 251-266.

2. W. Browder, Homotopy type of differentiable manifolds, Colloq. Algebraic Topology, Matematisk Institut, Aarhus Universitet, Aarhus, 1962, pp. 42-46.

3. A. Haefliger, Plongements différentiables de variettés dans variétes, Comment. Math. Helv. 36 (1961), 47-82. MR 26 \#3069.

4. - Knotted (4k-1)-spheres in 6k-space, Ann. of Math. (2) 75 (1962), 452466. MR $26 \# 3070$.

5. R. Lashof, Some theorems of Browder and Novikov on homotopy equivalent manifolds with an application, Notes, University of Chicago, Chicago, Ill. (note).

6. J. Levine, On differentiable imbeddings of simply-connected manifolds, Bull. Amer. Math. Soc. 69 (1963), 806-809. MR 27 \#5270.

7. - A classification of differentiable knots, Ann. of Math. (2) 82 (1965), 1550. MR $31 \# 5211$.

8. J. Milnor, Lectures on the h-cobordism theorem, Princeton Univ. Press, Princeton, N. J., 1965. MR 32 \#8352.

9. S. P. Novikov, Homotopically equivalent smooth manifolds. I, Izv. Akad. Nauk SSSR Ser. Mat. 28 (1964), 365-474; English transl., Amer. Math. Soc. Transl. (2) 48 (1965), 271-396. MR 28 \#5445.

Wesleyan University, Middletown, Connecticut 06457 